\title{
LE RECUL DE L'ESPACE AGRO-PASTORAL DANS LES PREALPES DU SUD ET SES CONSEQUENCES SUR LE PAYSAGE DE LA MOYENNE MONTAGNE ${ }^{1}$
}

\author{
G. A. DE Reparaz*
}

\begin{abstract}
RESUMÉ.- Dans les Prealpes du Sud, zone désertifiée aux très faibles densités $\left(5 \mathrm{~h} / \mathrm{km}^{2}\right)$, les friches héritées des anciennes zones cultivées ou des anciennes pâtures de l'agriculture traditionnelle, couvrent des espaces considérables (près de $60 \%$ du territoire). Les agriculteurs-eleveurs tres rares et vieillissants qui subsistent dans cet espace ne peuvent aménager ou entretenir celui-ci, et le saltus qui progresse encore actuellement prend des formes très diverses analysées ici. Une typologie communale évolutive des paysages de saltus, foncion de leurs origines diverses, est présentée. Les nouveaux habitants et les nouvelles fonctions de cet espace: tourisme "vert", résidences secondaires nombreuses, posent le problème des formes nouvelles de l'aménagement et de l'importance des acteurs extérieurs (Etat et reboisement massif, collectivités régionales, structures du tourisme).
\end{abstract}

ABSTRACT.-In the Southern Alps, a rural deeply depopulated region with very feeble densities (five inhabitants per $\mathrm{km}^{2}$ ) fallows inherited from the previously cultivated areas cover considerable surfaces -(almost $60 \%$ of the whole). The very rare agricultural sheep-farmers who still subsist in these areas have ground tooold to see to the upkeep of their holdings: so, the "saltus" which is still nowadays spreading on takes quite various forms analyzed in this article in a typology showing the evolution of the saltus landscape according of their varied origins.

The new inhabitants and the new functions of this space underline the problem of the new forms of agricultural management and of its external agents (State, Region and tourism structure).

RESUMEN.- En los Prealpes del sur, zona desertificada de muy bajas densidades $\left(5 \mathrm{~h} / \mathrm{km}^{2}\right)$, los eriales heredados de las zonas anteriormente cultivadas o pastadas en el marco de la agricultura tradicional ocupan una amplia extensión (cerca de $60 \%$ del territorio). Los agricultores -pastores, muy escasos, envejecidos, y en disminución, subsisten dificilmente en este

1 Reçu en Fevrier, 1992

* Institut de Géographie, 29 Av. R. Schuman F. 13621 AIX-EN-PROVENCE CEDEX France. 


\begin{abstract}
espacio; no pueden conservar ni gestionar adecuadamente el territorio rural. Los eriales, pues, siguen progresando en formas diversas, analizadas aqui, con una tipologia evolutiva de los saltus en las "communes".. según sus origenes.

Los nuevos habitantes y las nuevas funciones de este espacio: turismo "verde", segundas residencias numerosas, plantean el problema de las nuevas formas de la ordenación rural y de la importancia de los actores exteriores (Estado, región, reforestación masiva, estructuras del turismo).
\end{abstract}

Key words: agro pastoral space, fallow, saltus, desertification, postcultural environment, Southern Alps, middle mountain.

Partout, dans les montagnes européennes, la déprise agricole pose des problèmes majeurs d'environnement. Dans les montagnes françaises, le recul de l'emprise agricole a été deux fois plus rapide que dans la moyenne du pays durant les cinquante dernières années: alors que la surface agricole utilisée nationale perdait $14,5 \%$ entre 1939 et 1988 , elle diminuait de $28 \%$ dans les montagnes. Si l'on ajoute aux espaces agricoles proprement dits les prairies de fauche et les pâturages d'altitude régulièrement utilisés, ce

sont des pertes supérieures à $50 \%$ par rapport à 1939 que l'on constate dans les montagnes françaises en général. Mais le cas des moyennes montagnes méditerranéennes est bien plus sérieux encore. D'abord parce que le processus est beaucoup plus ancien et plus avancé qu'ailleurs, et que la déprise contemporaine touche au plus essentiel: les derniers ilôts agricoles et pastoraux. Ensuite parce qu'on aboutit ici à une véritable désertification humaine dans des montagnes moribondes d'un point de vue agricole, où les sociétés locales résiduelles ne peuvent plus entretenir qu'une partie très limitée des territoires communaux presque vides d'agriculteurs, avec bien moins d'un actif au $\mathrm{km}^{2}$. Les exploitants agricoles et éleveurs se comptent par unités et non plus par dizaines dans de très nombreuses communes péalpines. Orils sont les "producteurs" fondamentaux de l'espace humanisé, et ce sont eux qui maintiennent ce qui reste des paysages aménagés par des siècles de mise en valeur. Leur nombre de plus en plus réduit a entraîné un abandon progressif et grandissant de l'espace aménagé dans le cadre de la propriété privée, et ceci dans des territoires montagnards dont la fragilité est particulièrement grande.

Quelle est donc l'étendue, la part de ces espaces abandonnés très sensibles à l'érosion, à l'incendie, aux glissements de terrain, de ces friches, de ce saltus de substitution que s'étend sans cesse?

La nature même de cet espace pose évidemment aussi des problèmes très divers, car si la dégradation du milieu autrefois mis en valeur est une réalité écologique très fréquente (érosion, perte de diversité spécifique, disparition de biotopes, uniformisation, "fermeture" des paysages...), il n'est pas non plus rare de voir se développer un paysage "post agro-pastoral" qui revient lentement á un climax originel sous des formes plus favorables, et 
avec des aménagements partiels possibles dans un contexte de nouvelles utilisations de l'espace surtout par le tourisme et les loisirs.

La question du porquoi de cet abandon par paysans et bergers n'est pas, par ailleurs, sans importance, car selon les raisons et zones des abandons, des formes très distinctes de friches, d'ermes, se sont constituées, dont les conséquences paysagères diffèrent beaucoup, et dont le "traitement" éventuel par des efforts d'aménagement actuels devront tenir compte.

C'est, enfin, ce nouvel aménagement, les nouveaux usages, ou bien cette protection, - voire même l'abandon volontaire et plus ou moins raisonnéde l'espace délaissé par l'agriculteur-éleveur qui seront envisagés ici, plus particulièrement dans le cadre des Préalpes du Sud provençales. Le tourisme, les loisirs, la sylviculture et les diverses formes de reboisement, et parfois même la reprise extensive d'un élevage renouvelé, ont des impacts très différents sur l'environnement et, plus globalement sur les paysages au sens plein du terme, selon les formes particulières qu'ils prendront localement et selon les objectifs et les acteurs socio-économiques qui les animeront.

\section{Les Préalpes du Sud, un espace rural montagnard à l'évolution avancéé}

Les Préalpes du Sud -lato sensu-s'étendent sur quelque $180 \mathrm{~km}$., du massif du Cheiron aux abords de Nice jusqu'à l'extrémité occidentale des Baronnies au dessus de la vallée du Rhône, à l'Ouest. Nous n'envisagerons ici, essentiellement, que leur partie centrale: celle des Préalpes de Haute Provence (Préalpes de Digne, de Sisteron, de Castellane et du Verdon). C'est sans nul doute l'une des zones préalpines du Sud les plus caractéristiques et les plus vastes. Sur un peu plus d'une centaine de communes d'étude, s'étendant sur 333.000 ha, essentiellement dans le département des Alpes de Haute Provence, la densité moyenne est de 5,3 habitants au $\mathrm{km}^{2}$. Moyenne montagne aux très faibles densités, donc, et souvent atteinte par une véritable désertification: $26 \%$ des communes ont moins de $3 \mathrm{~h} . / \mathrm{km}^{2}$; 3 cantons (Turriers, Barrême, Noyers) ont $4 \mathrm{~h} . / \mathrm{km}^{2}$ et sur un total de 14 cantons 13 ont moins de $10 \mathrm{~h} . / \mathrm{km}^{2}$.

Mais ces chiffres, si expressifs soient-ils, n'expriment qu'une réalité globale, en partie corrigée par le fait que $85 \%$ des communes regagnent des habitants entre le recensement de 1982 et celui de 1990 , essentiellement par une variation positive du splde migratoire. Dans $81 \%$ des communes, en effet, on assiste à une légère mais réelle immigration alors que les soldes naturels restent presque partout négatifs. II s'agit donc là, pour plus des trois quarts des communes d'une désertification dépassée.

Mais ce changement démographique positif, déjà en partie amorcé entre 1975 et 1982, marque le début d'une profonde transformation socioéconomique car dans le même temps, la population active agricole proprement dite, déjà si peu nombreuse, continue au contraire de diminuer inexorablement. 


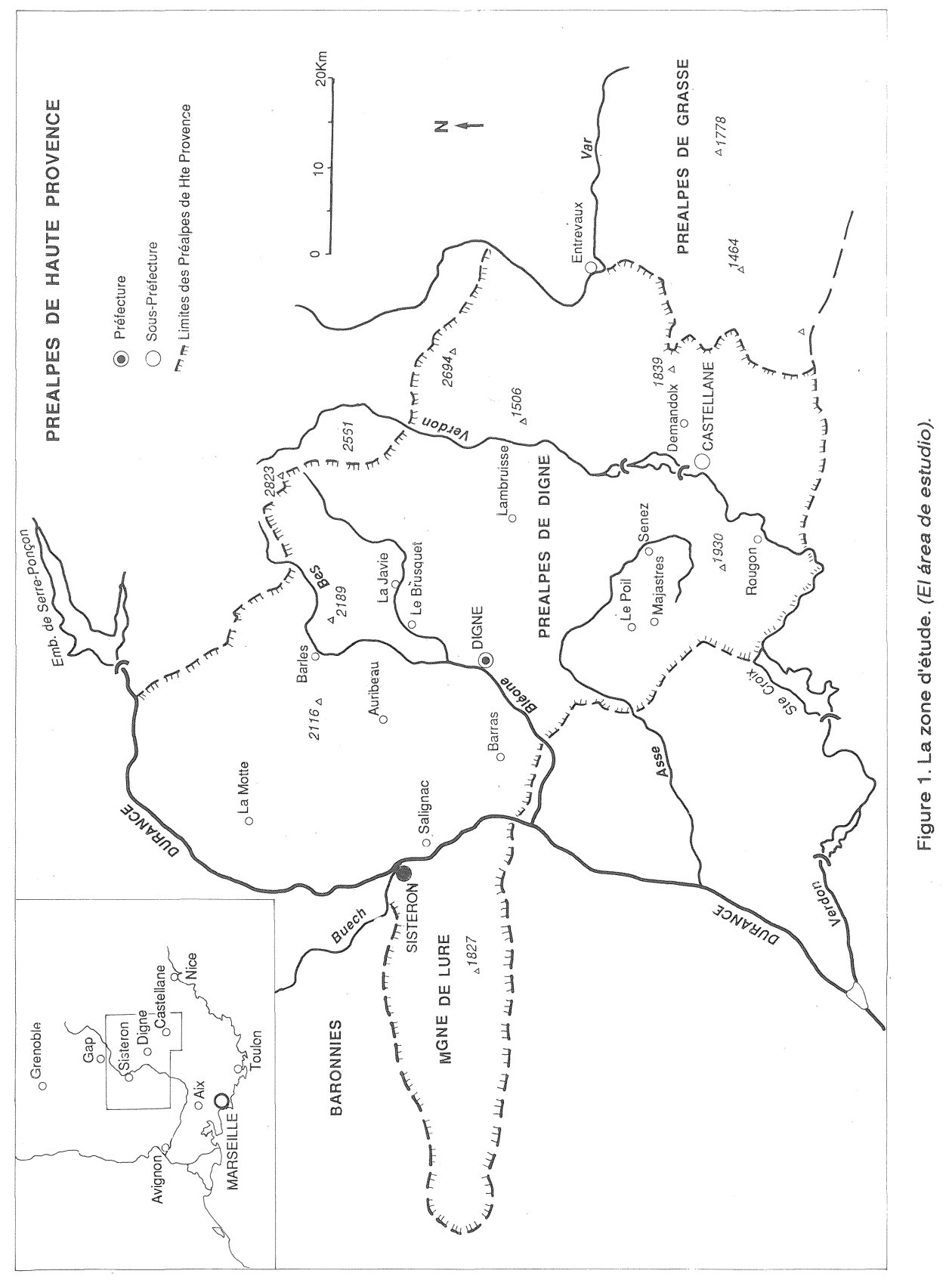

50 
RECUL DE L'ESPACE AGRO-PASTORAL

Sur 105 communes, 79 perdent des exploitants agricoles, 16 en restent au même nombre de paysans vieillissants, et seulement dix en ont légèrement gagné entre les recensements de l'agriculture de 1979 et de 1988 . Les producteurs et aménageurs de l'espace agropastoral continuent donc de disparaître dans les moyennes montagnes préalpines. Or, dans la plupart des communes, le nombre de cultivateurs-éleveurs est dramatiquement bas: dans 46 finages communaux on compte moins de 11 agriculteurs -7 communes en ont de 1 à 3 ! -et 50 communes, à peu près la moitié de l'échantillon, doivent se contenter de 11 à 25 agriculteurs... Encore s'agit-il, dans le compte fait par les statistiques du Ministère de l'Agriculture, de la totalité des exploitants agricoles, agriculteurs à temps partiel compris. Or ces derniers sont nombreux, exprimant par leur statut, même dans des zones très intérieures et isolées, un changement profond dans les combinaisons d'activités socio-économiques. Ils consacrent donc une part diminuée de leur travail à leur espaces productifs aménagés.

Dans la région agricole "Montagne de Haute Provence" du Ministere de l'Agriculture, qui comprend non seulement nos communes d'étude mais encore une vingtaine d'autres, plus montagnardes, $22 \%$ des exploitants agricoles étaient des agriculteurs consacrant la moitié ou plus de leur temps à une activité non agricole.

Moins de 1630 agriculteurs travaillent, au total, en 1988-89 dans un espace préalpin étudié dont on a vu qu'il s'étendait sur $3.330 \mathrm{~km}^{2}$ : à peine un agriculteur pour. $2 \mathrm{~km}^{2}$ d'espace rural. Ces chiffres doivent encore être nuancés négativement par le fait que parmi ces agriculteurs, produisant, aménageant et entretenant le territoire, il y a non seulement les nombreux actifs à temps partiel déjà évoqués, mais aussi surtout de nombreux hommes âgés.

Dans la "Montagne de Haute Provence", $37 \%$ des agriculteurs avaient plus de 60 ans. Les jeunes agriculteurs sont bien moins nombreux: 174 en tout, soit à peine $10 \%$. On peut estimer qu'à brève échéance, dans les 6 ou 8 prochaines années, à l'horizon 2.000, près de la moitié des exploitations n'aura pas de succession assurée dans cet espace difficile, montueux, cloisonné, très sensible à une érosion redoutable en raison de la fréquence des marnes et des roches tendres et peu cohérentes, desservi par un réseau routier étroit, d'un entretien coûteux, et donc d'une accessibilité difficile.

\section{Les friohes préalpines. Importance et nature d'un saltus omniprésent}

2.1. Lanótio pastoral montagnard, dans la moyenne montagne méditerranéenne, pose au départ un problème de définition.

La Commission des Communautés Européennes (CCE, 1979) donne la définition suivante: la friche "est une superficie antérieurement utilisée à des fins agricoles ou sylvicoles qui n'est plus exploitée et qui est recouverte toute 
"l'année de végetaux". Pour le Ministère de l'Agriculture français dont nous utiliserons les données statistiques, les "friches sont des superficies utilisables, mais non utilisées c'est à dire non comprises dans l'assolement agricole..." (de façon durable). Les friches peuvent ne présenter qu'un "caractère conjoncturel et provisoire" et les terres incultes, comme dans le cadre des "retraits" et du "gel" des terres CEE par exemple, peuvent être remises en culture si elles sont bien situées en zones planes de fond de vallée.

A ces friches conjoncturelles, voire parfois spéculatives, s'opposent les friches structurelles qui n'ont pratiquement aucune chance d'être réutilisées un jour pour un usage agricole. Curieusement les définitions officielles ne prennent pas en compte explicitement les friches issues de l'espace pastoral: prairies naturelles fauchées ou non, pâtures. C'est pourtant sur celles-cique s'est étendue et s'étend une partie particulièrement importante des friches montagnardes.

Friches d'anciens labours et friches d'anciennes prairies et pâtures sont caractérisées par une végétation spontanée présentant des stades de développement différents et variés.

En réalité, dans les montagnes préalpines comme dans toutes les montagnes méridionales européennes, il existe aussi toute une frange, avec de nombreuses transitions, entre surfaces sous-cultivées et surtout pâturages-pacages plus ou moins utilisés en temps et en charge animale, qui sont des "demi-friches", des "sous-pâturages" déjà en partie colonisés par la végétation spontanée inutilisable par l'élevage. Ces superficies intermédiaires sont en fait assez partiellement et diversement prises en compte, au niveau des statistiques agricoles, comme "Parcours et landes productifs" de la STH, dans les Recensements Généraux de l'Agriculture fondés sur les enquêtes exhaustives décennales des exploitations.

Dans ce cadre, une partie impossible à préciser des $57.000^{2}$ ha. de "Surface Toujours en Herbe" dans la Zone "Montagne de Haute Provence" et "Sisteronnais", est formée de "demi-friches" pastorales. II est d'ailleurs très remarquable de constater la diminution globale des STH entre les deux derniers recensements (1979 et 1988): elles perdent $50 \%$ de leur étendue dans le "Sisteronnais" (-6.000 ha:) et 17\% (-10.000 ha.) dans la "Montagne de Haute Provence". Ce qui a disparu est évidemment passé hors surface agricole utilisée, en friches essentiellement.

Une autre source permet d'apprécier l'étendue considérable des territoires non cultivés dans les montagnes préalpines, avec une incertitude moins grande quant à la différence entre l'utilisé ou utilisable, et le non utilisé et inutilisable. Cette source est le cadastre. Dans sa nomenclature, celui-ci comprend les "Landes", "auxquelles sont assimilés les terrains de faible valeur", non cultivés. Ces "Landes" sont très souvent explicitement divișées en "pâturages", en "vagues", et en "arides". Les "Landes-pâturages" sont

2 Soit $70 \%$ de la Surface Agricole Utile. 
précisément, pour l'essentiel; ces "demifriches" pastorales auxquelles nous faisions allusion. Les "vagues" sont au contraire, dans nos communes, des friches inutilisées, friches maigres à la végétation rare et coriace ou bien des friches "fermées" envahies par la broussaille, ou même constituées par des formations arbustives relativement homogenes: buxaies de Buxus sempervirens autrefois indiquées dans les cadastres "napoléoniens" des années 1830 sous le nom de "buissières" ou "boussières"; genêtières de Genista cinerea etc...

Dans les 106 cadastres communaux de la région des Préalpes de Digne étudiés par nous il y a une quinzaine d'années (DE RÉPARAZ, 1978) nous comptions au total 156.166 ha de "Landes" (pâtures, vagues et arides) soit $56 \%$ de l'espace total préalpin envisagé.

Plus les territoires communaux sont situés dans la zone méridionale, sèche, à nuance particulièrement méditerranéenne des Préalpes, plus la partie en Landes - pâturages", issue le plus souvent des anciennes terres cultivées et des anciens prés, est limitée, parsemée de prunneliers (Prunus spinosa), d'églantiers (rosae) et de genévries (Juniperus communis). Mais elle reste tout de même encore pâturée épisodiquement ou du moins pâturable. Au contraire la partie en Landes "vagues" est beaucoup plus étendue et bien moins végétalisée, avec des plages de terrain presque nu ou occupé partiellement par une végétation épineuse ou coriace de la strate herbacée prédominante. La partie en Landes - "arides" y est, enfin, inégalement importante, avec ses rochers, ses ravinements en "roubines", ses éboulis, ses lapiaz plus ou moins dévoloppés, ses rares touffes et arbustes résiduels. Un bon exemple est celui de la commune de Rougon ( $940 \mathrm{~m}$. au village, $3.487 \mathrm{ha}, 8$ à $900 \mathrm{~mm}$. de précipitations), dans la zone méridionale préalpine dominant les gorges du Verdon, avec ses 342 ha de Landes- "pâtures", ses 361 ha de Landes- "vagues" et ses 2.104 ha d" "arides" soit respectivement $10 \%, 10,3 \%$ et $60 \%$ du territoire communal. À l'inverse, au nord plus froid et humide de la région, une commune comme celle de Bayons ( $879 \mathrm{~m}$. au village, $6.310 \mathrm{ha}, 1.000 \mathrm{~mm}$.), comporte $1801 \mathrm{de}$ landes -"pâturages" où se mélangent anciennes terres et pâtures de qualité qui restent bien utilisées par l'élevage -elles sont même fait l'objet d'une restructuration foncière, -1701 ha de Landes- "vagues" et 364 ha d' "arides" (respectivement $28,5 \%, 26,09 \%$ et $5,8 \%$ de la surface totale communale).

La notion des "Landes" cadastrales doit aussi être complétée, pour définir l'ensemble du saltus postagricole et pastoral, par la prise en compte -très délicate-, mais inévitable, d'une partie des "bois taillis" des nomenclatures cadastrales. Celles-ci comprennent, en effet, des groupements arborés souvent clairsemés, mêlés de broussailles, "forêt claire qui passe progressivement aux fruticées" (DOUGUEDROIT, 1976). Pris ainsi dans son ensemble, le saltus, qui vient pour une part notable des anciennes terres et pâtures permanentes du maximum démographique du $1{ }^{\text {er }}$ tiers du $X I X^{\circ}$ siècle, occupe plus de 200.000 ha sur les quelque 300.000 ha de la région préalpine envisagée: $67 \%$ ! C'est dire son importance. 
Les incertitudes et imprécisions des informations cadastrales et agricoles pourrainet être corrigées par des analyses systématiques de photointerprétation et d'images de satellites comme cela a été réalisé ailleurs, au moins sur certains secteurs et thèmes (LASANTA 1988, 1989, 1990). Des études ponctuelles de ce type ont été par exemple réalisées par le CEMAGREF sur des secteus et parcelles expérimentaux. Par ailleurs, la difficulté d'études évolutives ne dispense pas de la précieuse référence historique récente aux plans et auxétats de sections cadastrales successives (1830-1914-1950).

\subsection{L'ampleur du recul des surfaces agro-pastorales et l'importance du saltus}

Ils sont partout impressionnants, avec, bien sûr, des nuances selon le type de zone et d'espace communal. Le recul a commencé très tôt: le maximum démographique des Préalpes du Sud se situe entre 1836 et 1841. Depuis, la montagne préalpine n'a cessé de perdre des hommes, jusqu'aux recensements de 1975 ou 80 . Mais siglobalement le nombre total d'habitants augmente à nouveau -modestement-il ne s'agit pas des actifs agricoles et de leur famille. Ceux-ci continuent au contraire actuellement de diminuer en nombre dans la plupart des communes préalpines: sur 106 communes, 79 perdent des agriculteurs (75\%), 16 restent stagnantes et seulement 10 se sont accrues de quelques agriculteurs entre 1979 et 1988 (Recensements' de l'Agriculture).

Sur une plus longue durée, dans la période récente (1970-1988), et globalement dans les deux grandes régions agricoles préalpines, le recul des producteurs-aménageurs fondamentaux de l'espace rural que sont les agriculteurs-éleveurs a continué de façon grave. (Tabl. 1).

TABLE 1

Nombre d'exploitations agricoles. (Número de explotaciones agricolas)

\begin{tabular}{|l|c|c|c|c|}
\hline Region agricole & R.G.A. 1970 & R.G.A. 1979 & R.G.A. 1988 & $\begin{array}{c}\text { Pertes: } \\
\text { N-\%. }\end{array}$ \\
\hline Sisteronnais & 585 & 516 & 394 & $\begin{array}{c}-241 \text { exp. } \\
-41 \%\end{array}$ \\
\hline $\begin{array}{l}\text { Montagne de Haute } \\
\text { Provence }\end{array}$ & 2.387 & 1.940 & 1.775 & $\begin{array}{c}-612 \text { exp. } \\
-26 \%\end{array}$ \\
\hline
\end{tabular}

Source: Recensements Généraux de la Population. 
Parallèlement, les surfaces agricoles sont maintenant très réduites et localisées à peu près exclusivement dans les fonds de vallées principales et secondaires, où elles se maintiennent tant bien que mal depuis une vingtaine d'années. En revanche le saltus et la forêt se sont considérablement étendus, recouvrant parfois presqu'entièrement des superficies communales à peu près complètement dépeuplées (tabl. 2: Majastres 98\%, Le Poil 99\%, Auribeau 91\%, Demandolx 90\%).

A lui seul, le saltus des friches embroussaillées, des genêtières, des formations herbacées dégradées, couvre en moyenne près des trois quarts des territoires communaux (74\%) dans les 104 communes étudiées.

La part et la nature variable du saltus déterminent des types zonaux et communaux différents.

Certaines zones de l'interieurdes massifs, avec des territoires communaux élevés, perchés sur des replats et pentes dominant les vallées principales, constituant des culs de sac en "bout de monde" très isolés, comportent une part considérable, voire presque totale de friches et terres "vagues": tel est le cas de la commune type de Majastres (tabl. 2): saltus d'abandon agricole à peu près total et d'abandon pastoral très avancé (il reste officiellement trois exploitations en 1988, avec 5 ha "cultivés", en cultures fourragères, et 460 brebis...), ceci dans un espace à peu près vide d'habitants permanents. A ce type d' "abandon agro-pastoral" en zone de très faible densité $\left(0,3 \mathrm{~h} . / \mathrm{km}^{2}\right.$ -10 habitants en 1990 sur $29,8 \mathrm{~km}^{2}$-) correspond vraiment le terme de désertification. Là, l'Etat surtout, et la collectivité régionale, sont pratiquement les seuls acteurs efficaces possibles pour la sauvegarde et l'aménagement de l'espace abandonné (pas de résidences secondaires dans la commune, d'accessibilité routière très médiocre). La reconstitution de la forêt, l'achat de foncier ou la restructuration foncière par remembrement "simplifié" y ont eu lieu. Une variante de ce type est constitué par le cas de Demandolx(e). Ici, la proximité de la minuscule sous-préfecture de Castellane (1.349 hab.) et la présence du lac de retenue avec usine hydroélectrique, de Chaudanne ont entraîné la présence d'habitants non-agricoles un peu plus nombreux ( $95 \mathrm{~h}$.,

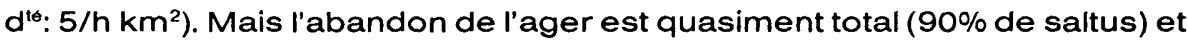
la forêt n'est absolument pas en cours de reconstitution: pas un hectare de forêt sur la commune. L'environnement est particulièrement délaissé et la fonction résidentielle reste à peu près la seule de la commune.

Un second type est constitué par de vastes territoires communaux de fond vallées terminales, adossées aux massifs, ou de portions de ces fonds de vallées très isolées, entre deux défilés en gorges, et comportant de très importantes surfaces en pré-pâtures d'altitude. Tel est le cas de Barles, établi entre ses deux "clues" où s'insinue, parfois en tunnels, une route récente, mais étroite et difficile. Là, depuis des siècles, l'espace-pacage a accueilli des transhumants et le saltus y est moins étendu qu'ailleurs (53\%) car les pâtures d'altitude, entre $1.100 \mathrm{~m}$. et $1.900 \mathrm{~m}$., continuent d'être en partie tondues par les bêtes, dans une situation non sans rapports avec le cas de Nocito, aux pieds de la Sierra de Guara, dans les Prépyrénées aragonaises. 
PIRINEOS 139

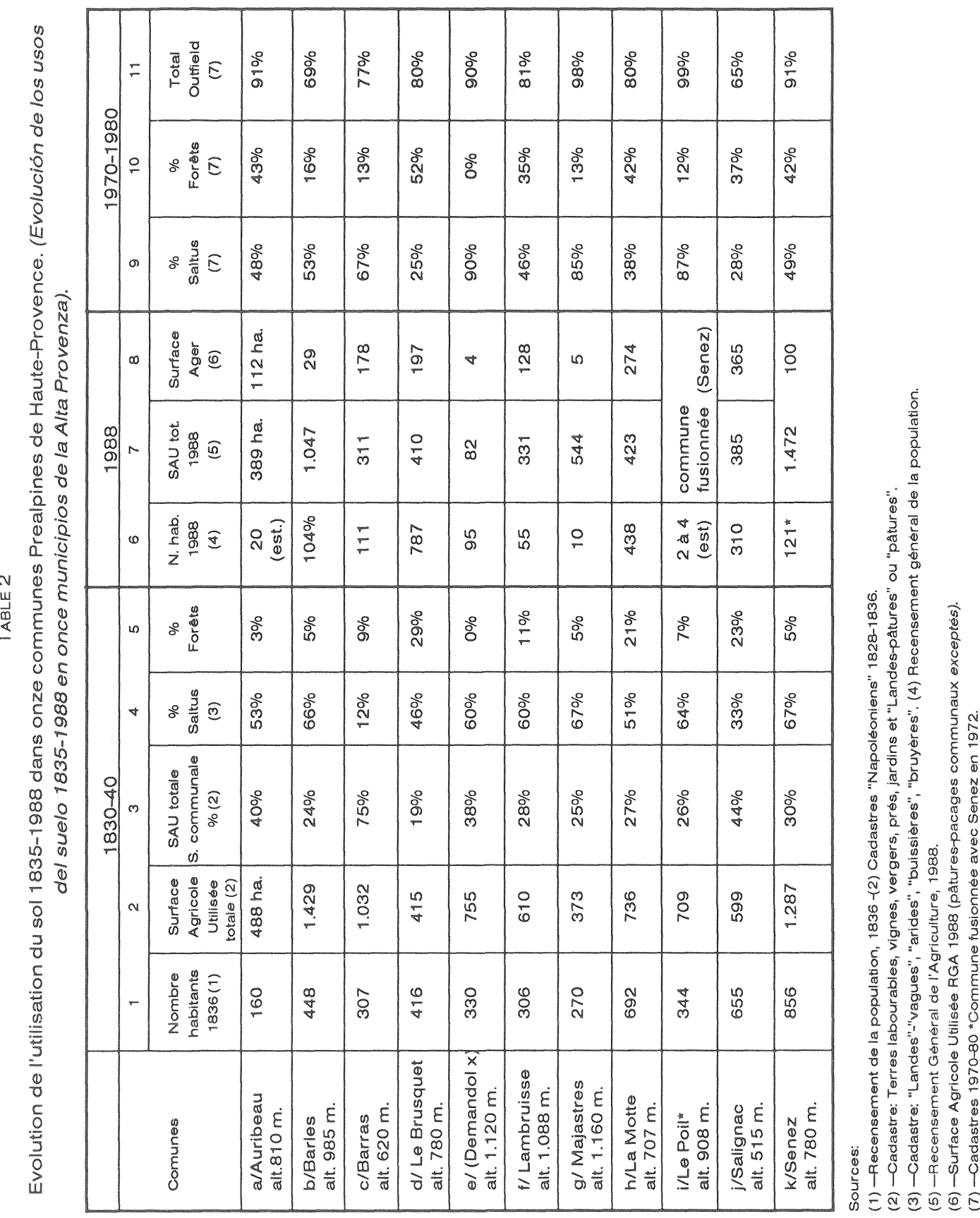

56 
RECUL DE L'ESPACE AGRO-PASTORAL

TABLE 3

Le saltus dans les communes des Prealpes de Hte Provence (1970-80). (El saltus en los municipios de la Alta Provenza, 1970-80)

\begin{tabular}{|c|c|c|c|}
\hline $\begin{array}{c}\text { \% de la surface } \\
\text { communale }\end{array}$ & $\begin{array}{c}\text { Nombre de } \\
\text { communes }\end{array}$ & Surface dans la commune & $\begin{array}{c}\text { Nombre de } \\
\text { communes }\end{array}$ \\
\hline - de $50 \%$ & 9 & - de 500 ha dans la \\
commune & 4 \\
\hline 50 à $60 \%$ & 4 & 500 à 1.000 ha & 30 \\
\hline 60 a $70 \%$ & 18 & 1.000 à 2.500 ha & 36 \\
\hline 70 à $80 \%$ & 28 & 2.500 à 4.000 ha & 26 \\
\hline 80 à $90 \%$ & 27 & Plus de 4.000 ha & 8 \\
\hline+ de $90 \%$ & 28 & & \\
\hline
\end{tabular}

Source: Cadastre, années 1970-80.

Saltus: "Landes", “vagues", "arides" des nomenclatures cadastrales.

Certains types de finages communaux de fonds de vallées plus amples, plus accessibles etplus peuplées se caractérisent par une certaine résistance agricole, avec une part moindre de saltus et par contre un effort de reboisement sur les anciennes zones de pacages ou d'agriculture abandonnées. Tels sont par exemple La Motte du Caire $(707 \mathrm{~m}$. alt., 16 h./ $\mathrm{km}^{2}$ ) avec $38 \%$ de friches et au contraire $42 \%$ de zones en forêts, ou bien encore Le Brusquet ( $25 \%$ de saltus) dans la moyenne vallée de la Bléone ( $780 \mathrm{~m}$. alt.), et Salignac ( $28 \%$ de saltus) sur le côté est du Val de Durance (515 $\mathrm{m}$. alt.). Communes presque périurbaines, les deux dernières aux abords de Digne et Sisteron, respectivement capitale départementale et ville de quelque importance. Les résidences de travailleurs urbains, avec de modestes migrations pendulaires, et de résidents secondaires touristiques y désorganisent en outre le foncier agricole et rural non cultivé, avec un saltus de "mitage" résidentiel.

\section{Aux origines des friches: causes, formes et moments de l'extension du saltus préalpin}

Les causes du développement des friches sont soit des causes anciennes, liées du vieux système agro-pastoral traditionnel, et maintenant dépassées, soit des causes anciennes mais toujours effectives, soit enfin des causes actuelles, directement en rapport avec les mécanismes socio-économiques contemporains. De là des friches anciennes et déjà très évoluées à côté de 
friches "jeunes", encore susceptibles de reconquête ou d'utilisation extensives nouvelles.

Par ailleurs, les origines des friches peuvent aussi être diverses par leur nature même: friches socio-démographiques, friches économiques, friches technologiques, friches structurelles foncieres...

Les plus anciennes friches, qui remontent au milieu du $X \mid X^{\circ}$ siècle, sont à la fois socio-démographiques et économiques: comme partout ailleurs, mais bien plus encore dans le milieu montagnard pauvre, les friches sont dues àl'abandon de terres marginales quiavaient été intégrées àl'ager sous la pression démographique, dans le cadre d'une économie familiale de subsistance. Avec la dépression démographique commencée dès 1840-50 -et qui continue toujours, avec d'ailleurs des paliers- les cultivateurs délaissent des ilôts plus ou moins vastes de terres de labour mises en valeur de façon souvent difficile et précaire dans des parties plus ou moins éloignées du village - l'habitat est surtout groupé ici-. Parfois pourtant ce sont aussi des fermes isolées, aventurées sur des replats élevés et ingrats qui sont abandonnées après une tentative de colonisation, généralement vers 1880-1900, ou après la Guerre de 1914-1918. La non-rentabilité de ces terres dans le cadre d'une économie plus ouverte et pour des agriculteurs moins nombreux explique ces abandons.

lly a par ailleurs le reculdans les espaces des pâtures-pacages: soit ceux situés aux confins de l'ager, soit ceux installés dans les zones hautes des communes: pâturages de moyenne montagne au dessus des forêts de chênes blancs-Quercus pubescens-et de fayards parfois-Fagus silvatica ou bien forêts de pins sylvestres. Ici, l'abandon est en rapport à la fois avec le recul d'un élevage dont l'importance relative était seulement due à l'addition de minuscules (de 5 a 15 ou 20 ovins...) mais nombreux troupeaux familiaux et avec la régression d'une transhumance "normale" des plaines provençales qui s'arrêtait en partie là aux temps d'une transhumance à pied moins exigeante.

Peu à peu, aussi, toutes les vieilles spécialisations régionales spéculatives d'une diversification agricole archaique mais rentable disparaissent: production de prunes por pruneaux ("pistoles"), d'amandes et de muriers séricicoles dans les zones basses, de fruits rustiques de longue conservation naturelle (poire "du curé") etc.... La crise céréalière de la fin du XIX ${ }^{\circ}$ siècle, la crise phylloxérique pour une partie non négligeable des communes préalpines, plus ou moins viticoles sur les bons adrets, se sont ajoutées à ces raisons socióéconomiques anciennes d'une première série d'abandons. Le manque de bras, dans une agriculture où une énorme consommation de travail était universelle, se fit beaucoup sentir dès 1880-1900 et alors que le progrès du nombre d'attelages de chevaux ou de mulets, et du train de culture en mécaniques spécialisées correspondantes, ne progressait que médiocrement.

L'isolement des villages perchés en "bouts de monde", réclamant désespérement durant des décennies des aides départementales pour 
RECUL DE L'ESPACE AGRO-PASTORAL

avoir une route carrossable, est une autre cause de l'abandon des ager et des espaces pastoraux. Dès la fin du siècle passé, certains de ces villages, les plus inacessibles, se dépeuplent totalement, ce qui est un record français, et nombre d'entre eux se videront encore, après la terrble saignée de la guerre de 1914-18, dont les effets on été particulièrement néfastes dans des villages montagnards déjà exsangues (Haut Val de Bés *, Haut Val de Bléone, Haute Asse ${ }^{3}$, Verdon). Des saltus "historiques", complexes et caractéristiques, constituent ainsi de vastes plages de friches autour de villages en ruines, avec des paysages autrefois humanisés qui gardent encore fortement l'empreinte émouvante de cette occupation antérieure malgré les colonisations végétales diverses qui les "ferment" maintenant. C'est lá d'ailleurs un des atouts actuels pour le nouveau tourisme "vert", patrimonial, archéologique et paysager.

Il faut ici constater -et c'est là un point essentiel-qu'il ne s'est créé à peu près nulle part, dans ces communes très isolées au degré d'accessibilité particulièrement difficile, des ensembles fonciers individuels agrandis, des unités économiques d'exploitation plus vastes et plus rentables constituées à partir des terres laissées vacantes par les émigrés ou les agriculteurs sans succession. En un mot, une concentration des exploitations, phénomene normal ailleurs, et engendrant des unités de production agricole qui auraient pu continuer la mise en valeur du territoire de ces communautés... II y a à ce problème deux explications essentielles. D'abord l'explication incontestablement déterministe: les terres et prés-pâtures laissés par les émigrés étaieric d'une qualité généralement si médiocre, et avec des potentialités si limitées, que certains, seulement ont été tout au plus loués, voire utilisés sans baux, et en général très partiellement, le reste allant à une friche définitive. Des ensembles économiques nouveaux, rentables dans un contexe général de plus en plus productiviste ne pouvaient donc être constitués que très exceptionnellement, avec, en plus, un parcellaire très exigu. D'autre part il y a eu un évident blocage foncier de la part de la diaspora des Préalpins. Plutôt que de vendre leurs terres -et leurs maisons de village, même en mauvais état-ils ont gardé pour la plupart, les cadastres ${ }^{4}$ en témoignent, leurs terres pendant parfois trois ou quatre générations, attachées à un bien familial en outre prudemment mis en réserve, et cela du dernier tiers du XIX ${ }^{\circ}$ siècle aux années 1940-50. Terres paralysées, donc, par ces types de relations sociologiques, psycho-sociologiques et socioéconomiques à la propriété.

3 -Tels Esclangon, Tanaron, Blégiers, Feissal, Trévans, Levens Creisset, Vière, Bédejun, Châteauneuf-les-Moustiers, Brandis, etc...: autant de communautés disparues et de communes supprimeés, aux finages à l'abandon.

4 -lls comportent, pour ces anciennes communes -et pour la plupart des autres- un nombre impressionnant de propriétaires dont, dès 1920-1930, beaucoup étaient déjà incertains, l'Etat faisant passer leurs biens aux domaines après un certain nombre d'années de non-paiement des impóts fonciers. 
Dans la période contemporaine les friches continuent de s'étendre un peu partout pour des raisons en partie nouvelles, outre le maintien des tendances antérieures. En premier lieu, on arrive dès 1965-70, à un contexte démographique très défavorable pour le groupe social des agriculteurs, a la fin de très nombreuses "exploitations-retraités"tenues par des exploitants de plus de 60 ou 65 ans, sans aucune succession assurée dans la très grande majorité des cas (tabl. 4).

TABLE 4

Importance des "exploitations-retraités" dans les cantons des Prealpes de HauteProvence en 1988 (exploitants de plus de 60 ans). (Importancia de los "jefes de explotación jubilados" en los cantones de los Prealpes de la Alta Provenza en 1988, agricultores de más de 60 años).

\begin{tabular}{|c|c|c|c|c|c|c|c|}
\hline Cantons & Annot & Barrême & Castellane & Entrevaux & La Javie & Mezel & $\begin{array}{l}\text { La Motte } \\
\text { du Caire }\end{array}$ \\
\hline $\begin{array}{l}\% \text { exploi- } \\
\text { tants }+ \text { de } \\
60 \text { ans }\end{array}$ & $45 \%$ & $35,5 \%$ & $46 \%$ & 53,5 & $26 \%$ & $40 \%$ & $28 \%$ \\
\hline Cantons & \multicolumn{2}{|c|}{ Noyers s/J } & \multicolumn{2}{|c|}{ St. André les A. } & \multicolumn{2}{|l|}{ Sisteron } & Turriers \\
\hline $\begin{array}{l}\% \text { expl. } \\
+60 \text { ans }\end{array}$ & \multicolumn{2}{|c|}{$34 \%$} & \multicolumn{2}{|c|}{$26 \%$} & \multicolumn{2}{|l|}{$36 \%$} & $25 \%$ \\
\hline
\end{tabular}

Source: Recensement Général de l'Agriculture 1988.

Plus du tiers des exploitations actuelles, en moyenne, avec des records de $45 \%$ et même $53 \%$ de celtes $-c i$, est donc condamné à très court terme, avec une extension corrélative de la friche sur une part probablement presque égale des terres et pâtures préalpines.

II faut ajouter à cela le problèmé de l" "abandon technologique". Déjà l'adoption généralisée du tracteur dès les années 60 avait obligé ál'abandon agricole de quantités de parcelles inaccessibles, ou trop en pente, ou trop petites et mal conformées pour l'usage de la machine, même quand il s'agissait des tracteurs de la première mécanisation (avec le tracteur léger à essence de 16 à 25-30 cv). On avait d'ailleurs tenté des expériences désastreuses, avec labours dans le sens de la pente, utilisations de charrues trop importantes et labours trop profonds, après l'araire et le cheval, et qui avaient conduit àl'abandon des champs dévastés par l'érosion. Actuellement il est encore moins possible de cultiver beaucoup d'anciens secteurs de l'ager avec le tracteur diesel de 35-50 cv et plus, et bien sûr d'y faire passer la moissonneuse -batteuse, la machine à couper la lavande, l'ensileuse ou la ramasseuse-presse à fourrage. L' "homme-machine" au tracteur ne peut 
RECUL DE L'ESPACE AGRO-PASTORAL

plus cultiver une grande partie des terrers que l'homme au cheval ou au mulet et au lichet ${ }^{5}$ mettait en valeur.

La friche sociale-structurelle de rétention foncière s'étend aussi de plus en plus, l'irruption du tourisme et de la résidence secondaire ayant des les années 60 profondément changé le rapport des propriétaires présents ou émigrés à la propriété du sol possédé. Le prix des parcelles autour des villages -les meilleures et les plus accessibles-, a en particulier considérablement augmenté, comme terres à bâtir potentielles ou effectives. II y $a$, et c'est essentiel, un nombre proportionnellement grandissant de résidences secondaires par rapport aux résidences permanentes dans les communespréalpines-jusqu'au double parfois-. Dans le seularrondissement de Castellane, l'un des plus pauvres des Préalpes, avec ses 32 communes toutes rurales, il y a 8.846 résidences secondaires contre 3.367 résidences principales occupées de façon permanente en 1990. Dans le lointain canton d'Entrevaux où tant d'agriculteurs sont des hommes ayant dépassé l'âge de la retraite, souvent célibataires, on trouve 537 résidences secondaires contre 468 résidences principales à la même date...

C'est dire que l'espace agro-pastoral préalpin est très fortement concurencé par un espace-loisirs-tourisme que la relative proximité d'une côte très urbanisée et la civilisation de l'automobile à l'échelle nationale-les résidences secondaires lyonnaises et parisiennes sont fréquentes dans les Préalpes- renforce encore. Dès les années 60, d'ailleurs, et de façon précoce, ce phénomène avait pris de l'ampleur: en 1962, l'arrondissement de Castellane -avec alors 47 communes- recelait 2.226 résidence secondaires contra 2.637 résidences principales, et 3.063 contre 2.647 en 1968. Et cela dans un espace aux services très limités et malgré un réseau routier étroit et difficile. En cela, les Préalpes du Sud diffèrent profondèment d'autres moyennes montagnes méditerranéennes, comme les Prépyrénées aragonaises par exemple, où le phénomène est infiniment moins développé, et de plus surtout actuellement limité à des résidences secondaires "retour" de résidents originaires des villages montagnards où ils ont réaménagé une maison et parfois remis en valeur les jardins irrigués périvillageois (dans certains villages du Serrablo, par exemple). À ce facteur touristique d'explication de la friche socio-culturelle par les résidences secondaires s'ajoutent d'autres facteurs-loisirs comme celui-quelque peu contradictoire -des friches "conservatrices" créées par une partie des agriculteurs et résidents locaux "du pays". II s'agit en particulier de terrains privés-ou communaux autrefois pâturés et employés collectivement-que la chasse, pratique essentiellement et jalousement locale, réserve-si elle ne les préserve pas toujours-, à la pratique de ce loisir du paysan ou de ses parents émigrés possédant biens et revenant à l'automne pour la pratiquer.

La friche purement économique est, enfin, une réalité contemporaine essentielle. Les deux principales ressourcesagricoles des Préalpes: l'élevage

5 -Pelle-bêche de bois chaussée de fer (laya esp.). 
ovin pour la viande d'agneau et la culture des lavandes, sont dans une situation de crise très grave. Plus de $60 \%$ des surfaces en lavande ont disparu dans le département des Alpes de Haute Provence entre 1958 et 1988 , essentiellement dans la zone préalpine où de très nombreuses communes n'en produisent plus sur les terrains difficiles et aux sols pauvres où elle avait été préférentiellement installée. Les cours à peu près constamment à la baisse depuis trente ans et la difficulté de la mécanisation en sont les causes principales. Les anciennes lavanderaies constituent donc, le plus souvent, maintenant, des friches ál'allure assez caractéristique, avec leur morphologie et leur cortège de plantes colonisatrices. Bien plus grave encore est la stagnation décennale, elle, des cours de la viande d'agneau, en raison de la concurrence britannique et néo-zélandaise ou des pays de l'Europe de l'Est. Ici, ce sont à peu près toutes les exploitations préalpines qui sont affectées, voire condamnées à court terme, malgré les primes de compensation et les indemnités spéciales montagne qui peuvent représenter jusqu'à quarante pour cent des revenus de l'élevage ovin "assité". Les espaces agricoles fourragers spécialisés (orge, légumineuses, prairies temporaires...) et surtout les prés et les pâtures-pacages d'intersaison ou d'été ont donc déjà considérablement diminué entre les recensements agricoles de 1970 et de 1988. La "Surface Toujours en Herbe" dans les exploitations agricoles a perdu, elle, 7.000 ha soit $50 \%$ de ses surfaces dans le Sisteronnais, et plus de 12.500 ha et $20 \%$ de son étendue dans la région agricole "Montagne de Haute Provence", entre ces deux dates. Friches en progrès notable, là encore, que rien ne semble devoir arrêter.

\section{Les paysages des terres abandonnées et leur évolution}

Globalement, les paysages des terres abandonnées varient d'abord suivant le degré d'ancienneté de leur abandon; ensuite suivant la nature de l'utilisation originelle du sol: labours, champs cultivés ou prés-pâtures, pacages; enfin, évidemment, selon la nature des terroirs oú se situaient les anciennes cultures ou pâtures (pentes, pédologie, hydrographie. exposition...). Mais en définitive, dans la définition des milieux géographiques variables où se développent ces friches, les éléments humains originels importent généralement davantage que les éléments naturels.

L'abandon, au XIX ${ }^{\circ}$ siècle, des terres de labours marginales, en pente ou en îôts de replats perchés, isolés, qui avaient été intégrées sous des pressions plus sociodémographiques qu'économiques, ont le plus souvent été, ensuite, et après une période transitoire ou non en pâtures inégalement dégradées, reboisées volontairement en pin noir d'Autriche surtout (Eaux et Forêts - ONF) ou naturellement, en pin sylvestre (si elles étaient restées propriété privée). Les plus exposées sont devenues la proie de l'érosion, parfois arrêtée par les efforts des services de Restauration des Terrains en Montagne (cas de Chaudon-Norante, R. Courtot et A. de RePARAZ, 1982). 
RECUL DE L'ESPACE AGRO-PASTORAL

Dans la période récente, les terres agricoles abandonnées au cours des vingt ou trente années évoluent diversement selon qu'elles sont sur des sols plus ou moins profonds et en pente, selon leur exposition, et selon qu'elles passent a l'abandon total directemente ou bien qu'elles connaissent une transition pastorale extensive et inégale en "prés-påtures" puis en "landespâtures". Nous avons vu, par exemple sur les cultures en terrasses périvillageoises des villages perchés en bouts du monde du secteur méridional des Préalpes ("Bloc de Majastres", bordures N du Verdon de Castellane à Moustiers...), une phase de transition en prés pâturés (en intersaison et en été) par des moutons venus de la vallée de l'Asse voisine, où étaient "descendus" s'installer des exploitants originaires des villages et communes "d'en haut". Actuellement, et surtout dans les adrets (solanas), une grande partie des anciens champs devenus prés sont envahis d'églantiers (Rosae), de prunelliers (Prunus spinosa) et d'aubépines (Crataegus monogina), dans un premier temps. Puis ce sont les genévriers (Juniperus communis) et surtout, presque partout, les genêts cendrés (Genista cynerea), plus rarement et localement les buis (Buxus sempervirens) et les amélanchiers. L'embroussaillement reste prépondérant sur ces adrets anciennement agricoles, avec une faible et lente progression du pin sylvestre, de l'érable champêtre (Acer campestre) et une strate herbacée en plages inégales où les brachypodes, (Brachypodium pinnatum), la lavande vraie, le serpolet (Thymus Serpillum), le lotier corniculé (Lotus corniculatus), les globulaires (Globularia cordifolia), l'héllébore fétide (Hellebours foetidus) sont les plantes les plus caractéristiques.

Aux ubacs (umbrias), les anciennes terres cultivées - plus rares- et surtout les anciens prés et pâtures "subissent les assauts de l'enrésinement (pin sylvestre) qui se propage à une vitesse étonnante par essaimage" (THÉPOT, 1977). La vitesse de croissance et de reboisement naturel du pin sylvestre nous paraît, en particulier dans les ubacs de la partie centrale et septentrionale des Préalpes, très supérieure à celle des Prépyrénées ou même des parties basses des chaînes méridionales pyrénéennes (observations T. LASANTA, 1991). En quinze à vingt ans, nous avons assisté à la colonisation à près complète et à la "fermeture" par boisement en pin sylvestre d'anciennes pâtures dans le secteur de St. André les Alpes.

En début de cycle de reconquête par la végétation naturelle, certaines zones d'ubacs en prairies anciennement irriguées souffrent beaucoup de l'abandon des réseaux de canaux et de rigoles d'irrigations échelonnés sur les pentes. Dans les parties hautes, ceux-ci, comblés, éventrés, déformés, constituent des points de départs d'érosion ou bien parfois des zones de mauvais drainage et écoulement, avec saturation et décollementsglissements de masses variables de terrains saturés.

Enfin, la vieille pratique de l'écobuage (artiga) et du feu pastoral pour maintenir les pacages ouverts et en état de pâturage, pratiquée avec expérience et profit toute l'année autrefois, puis seulement en hiver, est strictement interdite actuellement dans un espace beaucoup plus fermé et boisé et dans un contexte régional où le feu est devenu une hantise. 
La "fermeture" des paysages anciennement cultivés est donc une réalité de plus en plus générale, mais avec des nuances qui mettent en cause l'avenir des usages des espaces ruraux.

\section{Conclusions}

Friche érodée, friche arbustive, friche arborée et forêt, le vieil espace agropastoral transformé pose donc maintenant le problème de son nouvel aménagement pourdes populations permanentes ou saisonnières nouvelles.

Une première forme est évidemment le boisement ou reboisement systématique, déjà largement avancé sur des dizaines de milliers d'hectares d'anciennes terres et pâtures achetées par l'Etat depuis les années 18601880 "nis de 1860 et de 1882 permettant les expropriations pour cause d'utilité publique, parfois brutalement appliquées) et forêts communales "soumises" à sa gestion. Les crédits du Fonds Forestier National ont aussi encouragé certains propriétaires émigrés à reboiser sur des parcelles autrefois agro-pastorales.

C'est la solution de la "forêt partout", protectrice, patrimoniale, étatique, gérée par la collectivité nationale -l'O.N.F.- ou par des techniciens privés, et essentiellement destinée à des usages qui échappent aux habitants du pays, même si l'O.N.F. "ouvre" de plus en plus "ses" forêts au tourime vert, à la circulation auto -règlementée- et à pied, organise visites et stages malgré les reprises du braconnage observées (CHAPPAZ, 1989). Mais la forêt. c'est tout de même le désert humain la plus grande partie de l'année, et elle ne peut tout occuper, malgré les efforts de l'Office pour favoriser certaines expériences d'association de l'activité forestière et du pastoralisme extensif tel le pâturage sous forêt (Ch. BERTHET, 1980).

En ce qui concerne les friches-broussailles, il y a un peu partout dans les montagnes françaises des expériences de débroussaillement parfois réussies, aidées par des primes. Dans les Préalpes, des tentatives ont été faites dans ce sens et certains éleveurs continuent à pratiquer le surpâturage de débroussaillement en parcs (filets de plastique). Mais ce n'est là qu'une reconquête bien limitée de la friche pour un élevage renouvelé que la plupart des éleveurs ne peuvent se permettre. Ils ont, en effet, déjà misé sur en élevage intensifié, fondé sur une alimentation en parite basée sur l'achat d'aliments hors de l'exploitation et sur une agriculture "fourragère" mécanisée et endettée des fonds de vallée. Cependant un certain nombre de mesures et d'actions dont certaines en gestation peuvent permettre une réhabilitation et un aménagement renouvelé du paysage agro-pastoral: agriculture contractuelle d'entretien de l'espace, application réelle de la loi sur les terres incultes permettant des installations d'agriculteurs éleveurs extensifs, extérieurs à la région; développement des Associations Foncières Pastorales limitant l'anarchie stérilisante de la propriété cause de dégradation de l'espace rural, zonages de l'espace réservant le plus fragile à la protection, au boisement, mais le plus accesible et le moins menacé à l'agrotourisme et aux aménagements pastoraux-forestiers privés ou associatifs. 
L'espace loisirs-tourisme et l'espace patrimonial écologique préalpins ne peuvent en effet exister sans l'action de l'homme de la terre, agriculteur, éleveur, sylviculteur organisé coordonnant son activité avec les autres activités nouvelles organisatrices de l'espace.

\section{Bibliographie}

Archiloque, A.; Borel L. \& Devaux, J. P. (1974): Feuille d'Entrevaux au 1/50.000, in Bulletin de carte de la végétation de la Provence et des Alpes du Sud. Laboratoire de phytosociologie et cartographie végétale, pp. 87-129, Marseille.

BAZIN, G. etal. (1982): Propositions pour le développement de l'activité agricole dans les hautes valiées des Alpes du Sud. Rapport. Institut National de la Recherche Agronomique, 9 p., Paris.

BESSON, L. (1982): Rapport fait au nom de la Commission d'enquête sur la situation de l'agriculture et de l'économie rurale dans les zones de montagne et défavorisées . Rapport n. 757 à l'Assemblée Nationale, 406 p., Paris.

BERTHET-BONDET, Ch. (1980): Ateliers technologie et développement: compte rendu de l'atelier "Mise en valeur des espaces sylvo-pastoraux". Forêt Méditerranéenne, t. II, n. 2 , pp. 219-227. Marseille.

CALMES, R. \& FER, N. (1991): La dynamique des friches en pays d'Auge. In Colloque "Quelles campagnes pour demain", à paraître, 14 p., Rennes.

Chappaz, G. (1989): Contribution à l'étude de l'histoire agraire des Alpes du Sud. ADER, 73 p., Turriers.

COMMISSION DES COMMUNAUTES EUROPEENNES (1979): Conséquences écologiques de l'abandon des terres cultivées, in Information sur l'agriculture, $n .{ }^{9} 62$.

COMOLET, A. (1989): Prospective à long terme de la déprise agricole et environnement, Doct. de Synthèse, Institut pour une politique européenne de l'environnement, Ministère de l'Environnement, 166 p., Paris.

COURTOT, R. \& de Reparaz, A. (1982): Déprise agricole et mutations de l'organisation de l'espace dans les Préalpes du Sud: le cas de Chaudon-Norante. Méditerranée, n. 3 et 4 , pp. 71-76, Marseille.

Douguedrolt, A. (1976): Les paysages forestiers de Haute-Provence et des AlpesMaritimes, thèse d'Etat, $550 \mathrm{p}$. Edisud, Aix-en-Provence.

FAUDRY, D. et al. (1977): Désertification de l'espace montagnard, CETGREF-INERM, n. ${ }^{\circ}$ 11, 197 p., Grenoble.

GENARD, M. (1989): Comment étudier les conséquences écologiques de la déprise agricole; revue bibliographique. Bulletin Technique d'Information des ingénieurs agronomes, n. 436 , pp. 13-21, Paris.

LASANTA, T. (1988): The abandonment process of the cultivated space in Central Pyrénées. Pirineos, 132, pp. 11-36, Jaca.

LASANTA, T. (1989): Evolución reciente de la agricultura de montaña: el Pirineo aragonés. Geoforma Ediciones, 220 p., Logroño.

LASANTA, T. (1990): L'agriculture en terrasse dans les Pyrénées centrales espagnoles Méditerranée 3-4, tome 71, pp. 37-42, Aix.

MignON, Ch. (1991): La déprise agricole dans les montagnes françaises, in Colloque “Quelles campagnes pour demain”, à paraître, 11 p., Rennes.

Reparaz de, G. A. (1978): La vie rurale dans les Préalpes de Haute Provence. These d'Etat, 1.227 p., Edisud, Lille -Aix-en-Provence. 


\section{PIRINEOS 139}

Reparaz de, G. A. (1982): La montagne provençale investie; Provence Historique, tome XXXII, 130, pp. 391-407, Marseille.

Reparaz de, G. A. (1990): Le déclin de la lavande dans l'agriculture et dans la vie rurale de la Haute-Provence. Provence Historique, tome XL, 161, pp. 291-309, Marseille.

ТНЕРОт, N. (1977): Influence des processus socio-démographiques sur le système agraire dans la région des Préalpes de Digne, CTGREF-Fédération des procucteurs ovins des A.H.P., 46 p., Montpellier. 